\title{
Molecular detection of vector-borne agents in dogs from ten provinces of China
}

$\mathrm{Da} \mathrm{Xu}^{1 \dagger}$, Jilei Zhang ${ }^{1 \dagger}$, Zhengsheng Shi ${ }^{2}$, Chunlian Song ${ }^{3}$, Xiaofeng Zheng ${ }^{4}$, Yi Zhang ${ }^{5}$, Yongqing Hao ${ }^{6}$, Haiju Dong ${ }^{7}$, Lanjing Wei ${ }^{1}$, Heba S. El-Mahallawy ${ }^{1,8}$, Patrick Kelly ${ }^{9}$, Wenbin Xiong ${ }^{1}$, Heng Wang ${ }^{1}$, Jianji Li ${ }^{1}$, Xinjun Zhang ${ }^{1}$, Jianhong $\mathrm{Gu}^{1}$ and Chengming Wang ${ }^{1 *}$

\begin{abstract}
Background: Although many vector-borne agents are potential zoonoses and cause substantial morbidity and mortality in dogs worldwide, there are limited data on these organisms in dogs of China.

Methods: Quantitative PCRs for vector-borne agents were performed to investigate their prevalences in convenience whole blood samples obtained from 1114 dogs from 21 veterinary clinics and a commercial dog breeding facility in ten provinces of China. In addition, the PCRs were performed on 146 Rhipicephalus sanguineus senso lato and 37 Linognathus setosus collected from dogs in the commercial dog breeding facility.

Results: DNAs of Babesia gibsoni and B. vogeli (1.2\%), Ehrlichia canis (1.3\%), Hepatozoon canis (1.8 \%) and Theileria orientalis $(0.1 \%)$ or a closely related organism were detected in the bloods of the dogs studied, and Babesia vogeli (3.4\%) and Ehrlichia canis (4.1\%) in R. sanguineus senso lato. The qPCRs for Anaplasma spp., Dirofilaria immitis and Leishmania spp. were negative for all blood samples, ticks and lice. At least one vector-borne agent was found in dogs from 5 of the 10 provinces investigated in this study. Overall, $4.4 \%$ (49/1117) of the dogs studied were positive for at least one vector-borne agent with the prevalence being highest in the commercial breeding colony (24/97; $24.7 \%)$.
\end{abstract}

Conclusions: Our study confirms that B. vogeli, B. gibsoni, H. canis, and E. canis occur in China. Also, we present evidence that $T$. orientalis or a closely related organism can infect dogs.

Keywords: China, Vector-borne agents, FRET-PCR, Dogs

\section{Background}

Vector-borne diseases are important causes of morbidity and mortality in dogs and some have also emerged as a significant threat to human health worldwide [1-5]. Records of pet and guard dogs in China can be found dating 8000 thousand years ago and the current dog population is estimated to be between 150 and 200 million [6]. There is very little reliable information on vectorborne agents in dogs in China with only eight reports for the entire country: three using serology $(7,10,11)$, one using PCR (13), three using serology and PCR $(9,14,15)$, and one using PCR and microscopy (12). Studies with

\footnotetext{
* Correspondence: wangcm@yzu.edu.cn

${ }^{\dagger}$ Equal contributors

'Jiangsu Co-innovation Center for Prevention and Control of Important Animal Infectious Diseases and Zoonoses, Yangzhou University College of Veterinary Medicine, Yangzhou, Jiangsu 225009, P. R. China

Full list of author information is available at the end of the article
}

PCR and/or serology methods have indicated the E. canis occurs in dogs from Shenzhen, Guangzhou and Hong Kong [7-9] and D. immitis in dogs from Beijing, Shanghai, Chongqing, Yunnan, Guangdong and Shenyang provinces/ municipalities [7, 10, 11]. Molecular based studies have shown $D$. immitis in dogs from Liaoning province [12] and leishmaniasis to be common in dogs in Sichuan province [13-15]. Studies on ticks have revealed A. phagocytophilum in Haemaphysalis longicornis and Ixodes persulcatus from Suifenhe on the China-Russia border and Babesia spp. in dog ticks from six provinces/municipalities of China [16].

To provide further information on vector-borne agents in dogs in China, we investigated the prevalences of seven organisms (Babesia, Ehrlichia, Anaplasma, Dirofilaria immitis, Theileria, Hepatozoon and Leishmania) in blood samples from dogs in 10 provinces of China, and 
from ticks and lice collected from dogs in one province. Our findings are reported below.

\section{Methods}

\section{Collection of whole blood and external parasites}

Between November 2012 and February 2014, convenience whole blood samples were collected in EDTA by veterinarians who volunteered to participate (Table 1, Fig. 1). The dogs sampled in Taixing, Jiangsu province, were apparently healthy animals in a commercial dog breeding facility: convenience samples of ticks and lice were also collected from these dogs. The remaining dogs in the study were those attending 21 local veterinary clinics for routine health checks, vaccinations and for various conditions. Veterinarians were asked to comment on the presence or absence of ectoparasites on the dogs sampled.

Blood samples were frozen at $-20{ }^{\circ} \mathrm{C}$ before being put on ice (over 2 days) and sent to Yangzhou University College of Veterinary Medicine where they were frozen at $-80{ }^{\circ} \mathrm{C}$ until thawed at room temperature for DNA extraction. The external parasites collected from the dogs were identified following standard morphological criteria [17] and stored in vials with RNA/DNA Stabilization Reagent for Blood/Bone Marrow (Roche Diagnostics GmbH, Mannheim, Germany) at $-80^{\circ} \mathrm{C}$ until DNA extraction.

\section{Ethical approval}

The study was approved by the Institutional Animal Care and Use Committee of Yangzhou University, China. Written permission for sampling was obtained from the owners of dogs that participated in the study.

Table 1 The distribution of studied samples in ten provinces/ municipalities

\begin{tabular}{|c|c|c|c|c|}
\hline \multirow{2}{*}{$\begin{array}{l}\text { Sample } \\
\text { type }\end{array}$} & \multicolumn{4}{|l|}{ Source of samples } \\
\hline & Province/Municipality & City & Coordinates & Number \\
\hline \multirow[t]{12}{*}{ Dog blood } & Beijing & Beijing & $39^{\circ} \mathrm{N}, 116^{\circ} \mathrm{E}$ & 134 \\
\hline & Gansu & Lanzhou & $36^{\circ} \mathrm{N}, 103^{\circ} \mathrm{E}$ & 96 \\
\hline & Guangdong & Guangzhou & $23^{\circ} \mathrm{N}, 113^{\circ} \mathrm{E}$ & 35 \\
\hline & Henan & Zhengzhou & $34^{\circ} \mathrm{N}, 113^{\circ} \mathrm{E}$ & 102 \\
\hline & Inner Mongolia & Huhhot & $40^{\circ} \mathrm{N}, 111^{\circ} \mathrm{E}$ & 82 \\
\hline & Jiangsu & Yangzhou & $32^{\circ} \mathrm{N}, 119^{\circ} \mathrm{E}$ & 50 \\
\hline & & Taixing & $32^{\circ} \mathrm{N}, 120^{\circ} \mathrm{E}$ & 97 \\
\hline & & Nanjing & $32^{\circ} \mathrm{N}, 118^{\circ} \mathrm{E}$ & 130 \\
\hline & Shanghai & Shanghai & $31^{\circ} \mathrm{N}, 121^{\circ} \mathrm{E}$ & 84 \\
\hline & Shaanxi & Yangling & $34^{\circ} \mathrm{N}, 108^{\circ} \mathrm{E}$ & 56 \\
\hline & Xinjiang & Urumchi & $43^{\circ} \mathrm{N}, 87^{\circ} \mathrm{E}$ & 86 \\
\hline & Yunnan & Kunming & $25^{\circ} \mathrm{N}, 102^{\circ} \mathrm{E}$ & 162 \\
\hline Ticks & Jiangsu & Taixing & $32^{\circ} \mathrm{N}, 120^{\circ} \mathrm{E}$ & 146 \\
\hline Lice & Jiangsu & Taixing & $32^{\circ} \mathrm{N}, 120^{\circ} \mathrm{E}$ & 37 \\
\hline
\end{tabular}

\section{DNA extraction from whole blood, ticks and lice}

Aliquots $(200 \mu \mathrm{L})$ of whole blood were used for DNA extraction with High Pure PCR Template Preparation Kit (Roche Diagnostics GmbH, Mannheim, Germany) according to the manufacturer's instructions. The DNAs were eluted with $200 \mu \mathrm{L}$ Elution Buffer. Ticks and lice were individually homogenized in a shaker (Bertin Technologies, France) with four $3.0 \mathrm{~mm}$ ceramic beads for two periods of $15 \mathrm{~s}(3160 \times \mathrm{g}$ with a 15 -s break in between). DNAs were extracted from the homogenates with the QIAgen ${ }^{\circ}$ DNA Mini Kit (Qiagen, Valencia, CA, USA), eluted in $200 \mu \mathrm{L}$ of $1 \times \mathrm{T}_{10} \mathrm{E}_{0.1}$ buffer, and stored at $-80^{\circ} \mathrm{C}$ until PCR was performed.

\section{Quantitative FRET-PCRs}

Eight quantitative PCRs, seven for vector-borne agents and one for the mammalian HMBS gene as an endogenous internal control, were performed on a Roche LightCycler 480-II PCR Instrument. The qPCRs were performed as described previously for Anaplasma spp. [5], Babesia spp. [18], Dirofilaria immitis [19], Ehrlichia spp. [5], Hepatozoon spp. [20], Leishmania spp. [21], Theileria spp. [22] and the mammalian HMBS gene [23]. The positive PCR products were verified by gel electrophoresis and sequenced using forward and antisense primers (BGI, Shanghai, China).

\section{Standard PCR}

When $T$. orientalis was identified in the Theileria qPCR we used a standard PCR amplifying a 591 bp section (including 60-bp primers) of the 18S rRNA gene of Theileria spp. [22] to obtain longer sequences for analysis and further verification of identification.

\section{Results}

Information provided by veterinarians at the clinics where the study was performed indicated the vast majority of the dogs we sampled were pets that spent most of their time indoors. It was only very infrequently that the veterinarians noted ectoparasites on such dogs as they had very limited exposure to the outside environment and other animals. Ticks and lice were, however, found on the dogs in the Taixing commercial dog breeding facility which were housed more extensively. These were identified as Rhipicephalus sanguineus senso lato $(n=146)$ and Linognathus setosus $(n=37)$.

The qPCR for the HMBS gene was positive on all samples with $4.5 \times 10^{6} \pm 3.9 \times 10^{5}$ copies $/ \mathrm{ml}$ whole blood.

Overall, 4.4 \% (49/1117) of dogs were positive for at least one vector-borne agent and we obtained positive qPCR results for 4 of the 7 organisms we studied; there were only negative results for Anaplasma spp., D. immitis and Leishmania spp. At least one vector-borne agent was identified in 5 of the 10 provinces studied (Fig. 1). The 


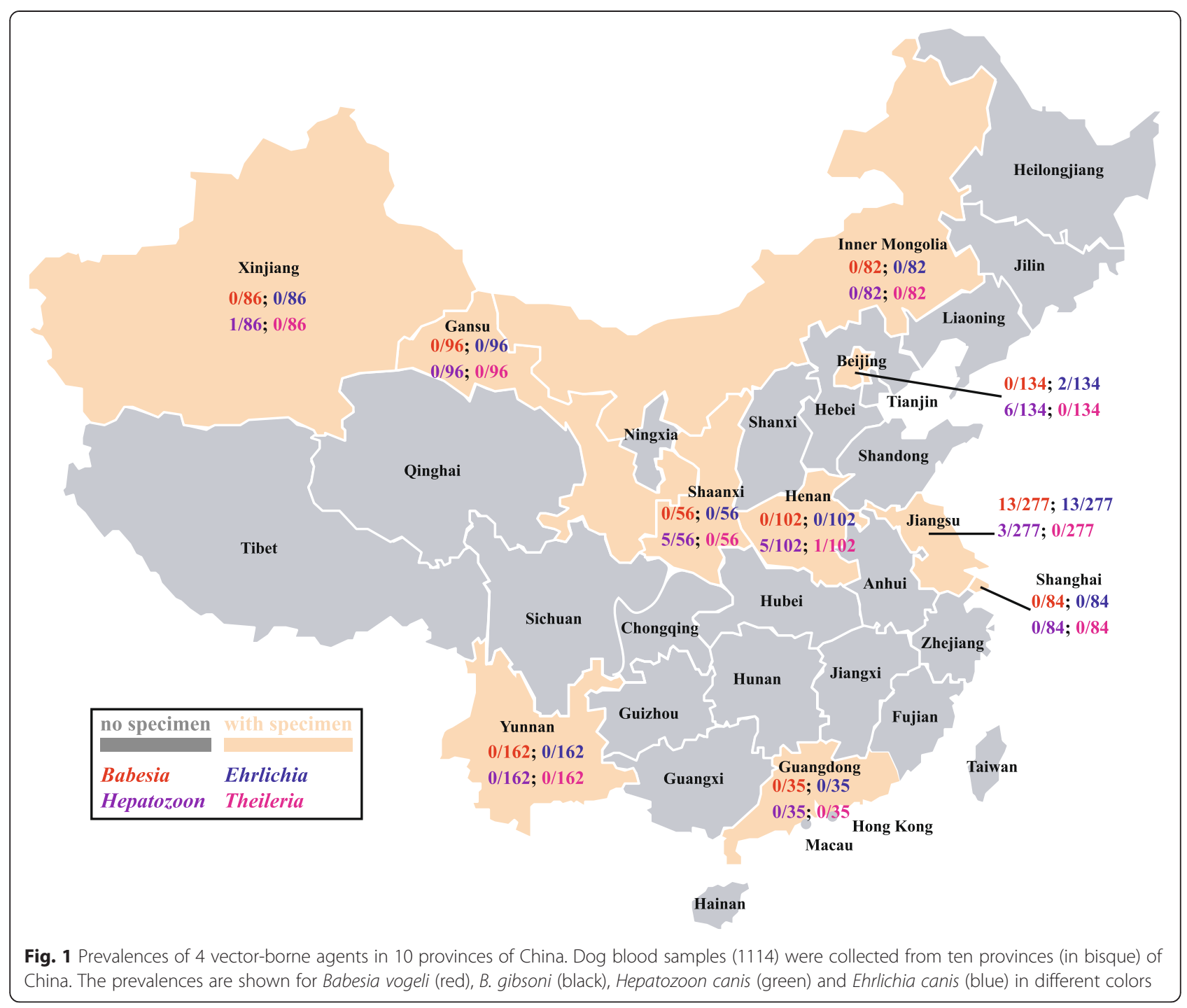

prevalence of vector-borne agents was highest in the dogs from the commercial breeding facility in Taixing (24/97, $24.7 \%)$ where external parasites were observed and collected. Six of the 146 (4.1\%) R. sanguineus senso lato collected were positive for $E$. canis but none of the L. setosus had evidence of a vector-borne agent (Tables 2 and 3 ).

The most common organism we identified was Hepatozoon canis with $1.8 \%$ (20/1114) of the dogs having PCR evidence of infection (Table 2). The prevalences of infection in Jiangsu $(1.1 \% ; 3 / 277)$ and Xinjiang provinces $(1.2 \%$; $1 / 86)$ were much lower than those found in Shaanxi $(8.9 \%$; 5/56), Henan (4.9\%; 5/102) and Beijing (4.9\%; 6/134). Genomic sequencing and BLASTN demonstrated that the partial 18S rRNA gene sequences for the $H$. canis we identified and deposited in GenBank database (Gene Accession \#: KP719091) were identical to one another, and also to that of a $H$. canis found in the spleen of a red fox in Austria (KM115995) (Table 3).
Overall, we found Ehrlichia canis DNA in $1.4 \%$ of the dog blood samples (15/1114) and $4.1 \%$ of the ticks (6/146). Ticks and most of the dogs positive for $E$. canis were from the Taixing commercial dog breeding facility (13/97 dogs, $13.4 \%$; 6/146 ticks, $4.1 \%)$. Only 2 of the positive dogs were from veterinary clinics (Beijing) where their clinical records showed them to be anemic. The sequences of the E. canis we found in the dogs and ticks (KP719093, KP719094) were identical to each other but showed 1 nucleotide mismatch with the sequence of the most closely related $E$. canis which was found in a dog in Japan (AF536827) (Table 3).

Thirteen dogs $(13 / 1114,1.2 \%)$ and 5 ticks (5/146, $3.4 \%$ ) were positive for Babesia spp. and sequencing showed two Babesia spp. occurred, most commonly B. vogeli (11 dogs and all ticks) followed by B. gibsoni $(2$ dogs). The sequences of the $B$. vogeli (KP719088, KP719089) from the dogs and ticks were identical but had 1 mismatch with the sequence of a 
Table 2 Prevalences of vector-borne agents detected by qPCR in dog bloods, ticks and lice in China

\begin{tabular}{|c|c|c|c|c|c|c|}
\hline \multirow{2}{*}{$\begin{array}{l}\text { Sample } \\
\text { type }\end{array}$} & \multicolumn{2}{|l|}{ Source of samples } & \multicolumn{4}{|c|}{ Percent (n) PCR positive } \\
\hline & Province/Municipality & City & Hepatozoon & Ehrlichia & Babesia & Theileria \\
\hline \multirow[t]{13}{*}{ Dog blood } & Beijing & Beijing & $4.5 \%(6 / 134)$ & $1.5 \%(2 / 134)$ & $0 \%(0 / 134)$ & $0 \%(0 / 134)$ \\
\hline & Gansu & Lanzhou & $0 \%(0 / 96)$ & $0 \%(0 / 96)$ & $0 \%(0 / 96)$ & $0 \%(0 / 96)$ \\
\hline & Guangdong & Guangzhou & $0 \%,(0 / 35)$ & $0 \%(0 / 35)$ & $0 \%(0 / 35)$ & $0 \%(0 / 35)$ \\
\hline & Henan & Zhengzhou & $4.9 \%(5 / 102)$ & $0 \%(0 / 102)$ & $0 \%(0 / 102)$ & $1.0 \%(1 / 102)$ \\
\hline & Inner Mongolia & Huhhot & $0 \%(0 / 82)$ & $0 \%(0 / 82)$ & $0 \%(0 / 82)$ & $0 \%(0 / 82)$ \\
\hline & \multirow[t]{3}{*}{ Jiangsu } & Yangzhou & $0 \%(0 / 50)$ & $0 \%(0 / 50)$ & $0 \%(0 / 50)$ & $0 \%(0 / 50)$ \\
\hline & & Taixing & $0 \%(0 / 97)$ & $13.4 \%(13 / 97)$ & $11.3 \%(11 / 97)$ & $0 \%(0 / 97)$ \\
\hline & & Nanjing & $2.3 \%(3 / 130)$ & $0 \%(0 / 130)$ & $1.5 \%(2 / 130)$ & $0 \%(0 / 130)$ \\
\hline & Shanghai & Shanghai & $0 \%(0 / 84)$ & $0 \%(0 / 84)$ & $0 \%(0 / 84)$ & $0 \%(0 / 84)$ \\
\hline & Shaanxi & Yangling & $8.9 \%(5 / 56)$ & $0 \%(0 / 56)$ & $0 \%(0 / 56)$ & $0 \%(0 / 56)$ \\
\hline & Xinjiang & Urumchi & $1.2 \%(1 / 86)$ & $0 \%(0 / 86)$ & $0 \%(0 / 86)$ & $0 \%(0 / 86)$ \\
\hline & \multirow[t]{2}{*}{ Yunnan } & \multirow[t]{2}{*}{ Kunming } & $0 \%(0 / 162)$ & $0 \%(0 / 162)$ & $0 \%(0 / 162)$ & $0 \%(0 / 162)$ \\
\hline & & & $1.8 \%(20 / 1114)$ & $1.3 \%(15 / 1114)$ & $1.2 \%(13 / 1114)$ & $0.1 \%(1 / 1114)$ \\
\hline Ticks & Jiangsu & Taixing & $0 \%(0 / 146)$ & $4.1 \%(6 / 146)$ & $3.4 \%(5 / 146)$ & $0 \%(0 / 146)$ \\
\hline Lice & Jiangsu & Taixing & $0 \%(0 / 37)$ & $0 \%(0 / 37)$ & $0 \%(0 / 37)$ & $0 \%(0 / 37)$ \\
\hline
\end{tabular}

B. vogeli reported in a dog from China (KJ939326). The B. gibsoni sequences (KP719090) were identical to each other and also to a B. gibsoni in a dog from Japan (LC012808) (Table 3).

A 5-year old, female Chihuahua dog with a perineal hernia seen in a veterinary clinic in Henan province was the only dog found to be positive for Theileria spp. The $18 \mathrm{~S}$ rRNA sequence of the Theileria spp. we identified in our $\mathrm{qPCR}$ was identical to that of $T$. orientalis
Thrissur 1 from India (KM609973) and Japan (XR 696404). Similarly, the sequence of the $591 \mathrm{bp}$ amplicon of the 18S rRNA gene we obtained with a subsequent standard PCR [22] was also identical to that of T. orientalis (KM609973, XR_696404).

Multiple infections with vector-borne agents were rare with two dogs and a tick having evidence of infection with $H$. canis and B. gibsoni, one dog with $E$. canis and $B$. vogeli, and one tick with $B$. vogeli and $E$. canis.

Table 3 Comparison of DNA sequences identified in this study with those having similar gene sequences in GenBank

\begin{tabular}{|c|c|c|c|c|c|}
\hline \multicolumn{3}{|c|}{ Isolates identified in this study } & \multicolumn{3}{|c|}{ Highly similar sequences in GenBank } \\
\hline Species & GenBank \# & Source & GenBank \# & Source & Mismatch \\
\hline \multirow[t]{4}{*}{ E. canis } & \multirow[t]{2}{*}{ KP719093 } & 13 dogs from Taixing & \multirow[t]{4}{*}{ AF536827 } & \multirow[t]{4}{*}{ Blood of dog from Kagoshima, Japan } & \multirow[t]{4}{*}{$1 / 158$} \\
\hline & & 2 dogs from Beijing & & & \\
\hline & \multirow[t]{2}{*}{ KP719094 } & 6 ticks (R. sanguineus senso lato) & & & \\
\hline & & from Taixing & & & \\
\hline \multirow[t]{3}{*}{ B. vogeli } & KP719088 & 11 dogs from Taixing & \multirow[t]{3}{*}{ KJ939326 } & \multirow[t]{3}{*}{ Blood of Springer Spaniel from Nanning, Guangxi, China } & \multirow[t]{3}{*}{$1 / 210$} \\
\hline & \multirow[t]{2}{*}{ KP719089 } & 5 ticks (R. sanguineus senso lato) & & & \\
\hline & & from Taixing & & & \\
\hline B. gibsoni & KP719090 & 2 dogs from Nanjing & LC012808 & Blood of dog from Yamaguchi, Japan & $0 / 210$ \\
\hline \multirow[t]{5}{*}{ H. canis } & \multirow[t]{5}{*}{ KP719091 } & 3 dogs from Nanjing & \multirow[t]{5}{*}{ KM115995 } & \multirow{5}{*}{$\begin{array}{l}\text { Spleen of infected Vulpes vulpes from Lower Austria, } \\
\text { Gaenserndorf, Austria }\end{array}$} & \multirow[t]{5}{*}{$0 / 144$} \\
\hline & & 6 dogs from Beijing & & & \\
\hline & & 5 dogs from Zhengzhou & & & \\
\hline & & 1 dogs from Urumchi & & & \\
\hline & & 5 dogs from Yangling & & & \\
\hline \multirow[t]{2}{*}{ T. orientalis } & \multirow[t]{2}{*}{ N/A } & 1 dog from Zhengzhou & \multirow[t]{2}{*}{ KM609973 } & \multirow[t]{2}{*}{ Blood of infected Bubalus bubalis from India } & $0 / 177$ \\
\hline & & & & & $0 / 591$ \\
\hline
\end{tabular}




\section{Discussion}

To date there have only been a few studies on vectorborne agents in Chinese dogs [7-9, 24, 25] but our study has confirmed that B. vogeli, B. gibsoni, E. canis, $H$. canis and $T$. orientalis or a closely related organism occur in China. The first three agents are very important pathogens of dogs and veterinarians in China should have an increased awareness of the possibility of infections in their canine patients and appropriate diagnostic tests and treatments should be made available. It is of note that the prevalences of infections were low in the dogs kept as companion animals and which were reported to seldom have ectoparasites. In contrast, ectoparasites were readily found on dogs from commercial dog breeding facilities and vector-borne agents were identified within these parasites. Veterinarians responsible for animals in such facilities should encourage tick control to prevent unnecessary morbidity and mortality. It should also be borne in mind that the prevalence and importance of vectors may vary considerably due to the influence of climactic and other environmental factors. China is a large country that can be divided into seven geographical regions. We studied dogs from each of these areas and found at least one vector-borne agent in five of the seven geographical regions. Future studies with more comprehensive and representative sampling should be performed to investigate the influence of climactic and environmental impact on the distributions of vectors and their agents in the different regions of China.

Hepatozoon canis was the most common vector-borne agent we identified and also the most widespread, being identified in dogs from 5 of the 10 provinces we studied. The organism also occurs widely around the world and, although moderate to severe disease can occur [26], most infections are sub-clinical.

The short (177 bp) and long (591 bp) 18S rRNA nucleotide sequences that we obtained for the Theileria sp. we identified were identical to that of $T$. orientalis from India (KM609973) and Japan (XR_696404). Theileria orientalis is normally found in yaks (Bos grunniens), cattle and buffaloes (Bos bubalis) and is transmitted by Haemaphysalis spp. [27, 28]. To the best of our knowledge, ours is also the first report that $T$. orientalis or a closely related organism might occur in dogs. How dogs become exposed to this organism in China and the pathogenicity and significance of infections requires further investigation.

Although Wang and Zhang et al. were unsuccessful in identifying $E$. canis infections in dogs in China using serology and PCR $[29,30]$, other workers have found serological and molecular evidence of infections in the blood of $2 \%$ of dogs in Shenzhen [7-9] and the organism in $R$. sanguineus senso lato ticks from dogs in China [31]. We have now found PCR evidence that $E$. canis infections are relatively common in Taixing, Jiangsu province. Canine ehrlichiosis is a common disease of dogs around the world that is a cause of considerable morbidity and mortality [32]. There are no vaccines available and treatment can be problematic. The disease is best combated by preventing infections with appropriate tick control strategies.

Our findings of B. vogeli and B. gibsoni at relatively low levels in dogs from Taixing and Nanjing, Jiangsu province, is consistent with a previous report that levels of infection with Babesia spp. are low in domestic dogs in China [24]. The relatively low percentage $(3.4 \%)$ of ticks we found with $B$. vogeli in Taixing, Jiangsu province, was similar to that reported in other provinces, mainly Guangdong (3.6 \%; 1/28), Hainan (3.3\%; 4/121) and Zhejiang $(6.7 \% ; 1 / 15)$ [16]. It was, however, lower than that reported in Chongqing $(25.0 \% ; 4 / 16)$ and Guangxi $(12.5 \%$; 11/88), indicating there is considerable regional variation in infection rates in China. While $B$. vogeli infections can cause severe disease in puppies, greyhounds and immune-suppressed dogs, infections usually only result in mild signs or are subclinical [4, 33, 34], $B$. gibsoni on the other hand is generally regarded as being more pathogenic and can cause severe disease which responds poorly to drug therapy [35]. There are no vaccines and prevention depends on adequate tick control and, as B. gibsoni can be transmitted in blood and by bites during dog fights [4].

We were unable to identify Anaplasma spp., Dirofilaria spp. and Leishmania spp. in our study. This is in contrast to earlier reports from China where $A$. phagocytophilum was demonstrated by PCR in dog ticks from Suifenhe, Heilongjiang province (5.9 \%), and in dog blood samples from nine provinces of China (10.9\%; 11 / 102) [36, 37]. Further, D. immitis infections have been demonstrated by microscopic examination and PCR test in dogs from Dandong, Liaoning province (24.0 \%; 147/ 886), and Leishmania spp. demonstrated by real-time PCR test in dogs $(24.8 \%$; 78/314) in Sichuan province [12, 13, 36, 37]. While the different observations might have been due to regional differences in infection rates, it is most likely they were due to demographical differences with the dogs in the above studies being more free-ranging and less well cared for and hence more likely to be exposed to ectoparasites. Larger and more inclusive studies are indicated to more accurately determine the prevalences and distribution of vector-borne agents in dogs in China.

\section{Conclusions}

In summary, we found the DNAs of Babesia gibsoni and B. vogeli (1.2 \%), Ehrlichia canis (1.3 \%), Hepatozoon canis (1.8\%), and Theileria orientalis $(0.1 \%)$ in the bloods of the dogs studied. Further, we found Babesia 
vogeli (3.4\%) and Ehrlichia canis (4.1\%) in the R. sanguineus senso lato. Our data from 10 provinces in China show a wide range of important vector-borne pathogens occur in dogs and further larger scale studies are indicated to determine more accurate prevalence data for these agents.

\section{Competing interests}

The authors declare that they have no competing interests.

\section{Authors' contributions}

WC and ZJ participated in the design of the study and performed the statistical analysis. ZJ and XD carried out the experiments. SZ, SC, ZX, ZY, HY, $\mathrm{DH}, \mathrm{WL}$, El-Mahallawy. HS, XW, WH, LJ, ZX KP, and GJ participated in the samples' collection. WC, KP and ZJ drafted the manuscript. All authors read and approved the final manuscript.

\section{Acknowledgments}

This project was supported by grants from the National Natural Science Foundation of China (NO: 31272575) and the Priority Academic Program Development of Jiangsu Higher Education Institutions, Yangzhou, Jiangsu, P. R. China.

\section{Author details \\ ${ }^{1}$ Jiangsu Co-innovation Center for Prevention and Control of Important Animal Infectious Diseases and Zoonoses, Yangzhou University College of Veterinary Medicine, Yangzhou, Jiangsu 225009, P. R. China. ${ }^{2}$ China Agricultural University College of Veterinary Medicine, Beijing 100083, China. ${ }^{3}$ Yunnan Agricultural University College of Animal Science \& Technology, Kunming, Yunnan 650201, China. ${ }^{4}$ Jiangsu Agri-animal Husbandry Vocational College, Taizhou, Jiangsu, China. ${ }^{5}$ Xinjiang Agricultural University College of Veterinary Medicine, Urumchi, Xinjiang 830052, China. ${ }^{6}$ Inner Mongolia Agricultural University College of Veterinary Medicine, Hohhot, Inner Mongolia 010018, China. ${ }^{7}$ Henan Agricultural University College of Animal Science and Veterinary Medicine, Zhengzhou, Henan 45002, China. ${ }^{8}$ Faculty of Veterinary Medicine, Suez Canal University, Ismailia 41522, Egypt. ${ }^{9}$ Ross University School of Veterinary Medicine, St. Kitts \& Nevis, West Indies.}

Received: 27 August 2015 Accepted: 28 September 2015

Published online: 01 October 2015

\section{References}

1. Groves MG, Dennis GL, Amyx HL, Huxsoll DL. Transmission of Ehrlichia canis to dogs by ticks (Rhipicephalus sanguineus). Am J Vet Res. 1975;36:937-40.

2. Harrus S, Waner T. Diagnosis of canine monocytotropic ehrlichiosis (Ehrlichia canis): an overview. Vet J. 2011;187:292-6.

3. O'Dwyer LH. Brazilian canine hepatozoonosis. Rev Bras Parasitol Vet. 2011:20:181-93.

4. Solano-Gallego L, Baneth G. Babesiosis in dogs and cats-expanding parasitological and clinical spectra. Vet Parasitol. 2011;181:48-60

5. Kelly PJ, Xu C, Lucas H, Loftis A, Abete J, Zeoli F, et al. Ehrlichiosis, babesiosis, anaplasmosis and hepatozoonosis in dogs from St. Kitts, West Indies. PLoS One. 2013;8:e53450.

6. Ma D, Ding X, Cao J, Xun X, Cheng Z. The situation of dog source in China. China Working Dog. 2012;1:45-50.

7. Xia Z, Yu D, Mao J, Zhang Z, Yu J. The occurrence of Dirofilaria immitis, Borrelia burgdorferi, Ehrlichia canis and Anaplasma phagocytophium in dogs in China. J Helminthol. 2012;86:185-9.

8. Hua P, Yuhai M, Shide T, Yang S, Bohai W, Xiangrui C. Canine ehrlichiosis caused simultaneously by Ehrlichia canis and Ehrlichia platys. Microbiol Immunol. 2000;44:737-9.

9. Wong SS, Teng JL, Poon RW, Choi GK, Chan KH, Yeung ML, et al. Comparative evaluation of a point-of-care immunochromatographic test SNAP 4Dx with molecular detection tests for vector-borne canine pathogens in Hong Kong. Vector Borne Zoonotic Dis. 2011;11:1269-77.

10. Sun M, Zhuo W, Guo S, Liao S, Shi D, Liu J, et al. Serological survey of canine dirofilariosis in Chongqing, Kunming, Nanchang, Fuzhou, Guangzhou, Shenzhen, and Nanning in Southern China. Vet Parasitol. 2012;185:225-8
11. Liu C, Yang N, He J, Yang M, Sun M. Prevalence of Dirofilaria immitis in dogs in Shenyang, Northeastern China. Korean J Parasitol. 2013;51:375-7.

12. Hou H, Shen G, Wu W, Gong P, Liu Q, You J, et al. Prevalence of Dirofilaria immitis infection in dogs from Dandong, China. Vet Parasitol. 2011;183:189-93.

13. Shang LM, Peng WP, Jin HT, Xu D, Zhong NN, Wang WL, et al. The prevalence of canine Leishmania infantum infection in Sichuan Province, southwestern China detected by real time PCR. Parasit Vectors. 2011:4:173.

14. Wang JY, Ha Y, Gao CH, Wang Y, Yang YT, Chen HT. The prevalence of canine Leishmania infantum infection in western China detected by PCR and serological tests. Parasit Vectors. 2011;4:69.

15. Sun K, Guan W, Zhang JG, Wang YJ, Tian Y, Liao L, et al. Prevalence of canine leishmaniasis in Beichuan County, Sichuan, China and phylogenetic evidence for an undescribed Leishmania sp. in China based on 7SL RNA. Parasit Vectors. 2012;5:75.

16. Wei FR, Lan QX, Zhu D, Ye JH, Liu Q, Zhang Y. Investigation on Babesia in ticks infested on police dogs in selected areas of China. Zhong Guo Ji Sheng Chong Xue Yu Ji Sheng Chong Bing Za Zhi. 2012;30:390-2.

17. CDC. Pictorial keys to arthropods, reptiles, birds, mammals of public health significance. 2013. www.cdc.gov/nceh/ehs/Publications/Pictorial_Keys.htm. (assessed 10 January 2015).

18. Wang C, Ahluwalia SK, Li Y, Gao D, Poudel A, Chowdhury E, et al. Frequency and therapy monitoring of canine Babesia spp. infection by high-resolution melting curve quantitative FRET-PCR. Vet Parasitol. 2010;168:11-8.

19. Thanchomnang $T$, Intapan PM, Lulitanond V, Sangmaneedet $S$, Chungpivat S, Taweethavonsawat P, et al. Rapid detection of Dirofilaria immitis in mosquito vectors and dogs using a real-time fluorescence resonance energy transfer PCR and melting curve analysis. Vet Parasitol. 2010;168:255-60.

20. Li Y, Wang C, Allen KE, Little SE, Ahluwalia SK, Gao D, et al. Diagnosis of canine Hepatozoon spp. infection by quantitative PCR. Vet Parasitol. 2008; 157:50-8.

21. Tsukayama P, Núnez JH, De Los Santos M, Soberón V, Lucas CM, Matlashewski G, et al. A FRET-based real-time PCR assay to identify the main causal agents of New World tegumentary leishmaniasis. PLoS Negl Trop Dis. 2013;7:e1956.

22. Yang Y, Mao Y, Kelly P, Yang Z, Luan L, Zhang J, et al. A pan-Theileria FRET-qPCR survey for Theileria spp. in ruminants from nine provinces of China. Parasit Vectors. 2014;7:413.

23. Wei L, Kelly P, Zhang J, Yang Y, Zheng X, Tao J, et al. Use of a universal hydroxymethylbilane synthase (HMBS)-based PCR as an endogenous internal control and to enable typing of mammalian DNAs. Appl Microbiol Biotechnol. 2014;98:5579-87.

24. Shen Y, Gao J, Xu K, Xue L, Zhang Y, Shi B, et al. Babesia in Nanjing area, China. Trop Anim Health Prod. 1997;29(4 Suppl):19S-22.

25. Chen Z, Liu Q, Jiao FC, Xu BL, Zhou XN. Detection of piroplasms infection in sheep, dogs and hedgehogs in Central China. Infect Dis Poverty. 2014;3:18.

26. Baneth G. Perspectives on canine and feline hepatozoonosis. Vet Parasitol. 2011;181:3-11. doi:10.1016/j.vetpar.2011.04.015. Epub 2011 Apr 19.

27. Fujisaki K, Kawazu S, Kamio T. The taxonomy of the bovine Theileria spp. Parasitol Today. 1994;10:31-3.

28. Yin H, Luo J, Schnittger L, Lu B, Beyer D, Ma M, et al. Phylogenetic analysis of Theileria species transmitted by Haemaphysalis qinghaiensis. Parasitol Res. 2004:92:36-42

29. Wang S, He J, Zhang L. Serological investigation of vector-borne disease in dogs from rural areas of China. Asian Pac J Trop Biomed. 2012:2:102-3.

30. Zhang XC, Zhang LX, Li WH, Wang SW, Sun YL, Wang YY, et al. Ehrlichiosis and zoonotic anaplasmosis in suburban areas of Beijing, China. Vec Borne Zoonotic Dis. 2012;12:932-7.

31. Wen B, Cao W, Pan H. Ehrlichiae and ehrlichial diseases in China. Ann N Y Acad Sci. 2003;990:45-53.

32. Little SE. Ehrlichiosis and anaplasmosis in dogs and cats. Vet Clin North Am Small Anim Pract. 2010;40:1121-40.

33. Cacciò SM, Antunovic B, Moretti A, Mangili V, Marinculic A, Baric RR, et al. Molecular characterization of Babesia canis canis and Babesia canis vogeli from naturally infected European dogs. Vet Parasitol. 2002;106:285-92.

34. Salem NY, Farag HS. Clinical, hematologic, and molecular findings in naturally occurring Babesia canis vogeli in Egyptian dogs. Vet Med Int. 2014;2014:270345. 
35. Ayoob AL, Hackner SG, Prittie J. Clinical management of canine babesiosis. J Vet Emerg Crit Care (San Antonio). 2010;20:77-89.

36. Zhang L, Liu H, Xu B, Lu Q, Li L, Chang L, et al. Anaplasma phagocytophilum infection in domestic animals in ten provinces/cities of China. Am J Trop Med Hyg. 2012;87:185-9.

37. Jiang JF, Jiang BG, Yu JH, Zhang WY, Gao HW, Zhan L, et al. Anaplasma phagocytophilum infection in ticks. China-Russia border Emerg Infect Dis. 2011;17:932-4.

Submit your next manuscript to BioMed Central and take full advantage of:

- Convenient online submission

- Thorough peer review

- No space constraints or color figure charges

- Immediate publication on acceptance

- Inclusion in PubMed, CAS, Scopus and Google Scholar

- Research which is freely available for redistribution 